\title{
Apolipoprotein e4 allele and endothelium-dependent arterial dilation in Type 2 diabetes mellitus without angiopathy
}

\author{
X. Guangda, W. Yuhua \\ Department of Endocrinology, Wuhan General Hospital, Wuhan, People's Republic of China
}

\begin{abstract}
Aims/hypothesis. Several studies have suggested a predisposing role of the $e 4$ allele of apolipoprotein $\mathrm{E}$ (ApoE) in the development of atherosclerosis and cardiovascular disease in Type 2 diabetes. Therefore, we hypothesized that the $e 4$ allele is also a risk factor for endothelial dysfunction. We attempted to assess whether Apo e4 allele is associated with endothelial dysfunction in the early stage of Type 2 diabetes.

Methods. We selected 255 Chinese Han Type 2 diabtetic men without angiopathy. PCR or allele-specific oligonucleotide probes were used to analyse ApoE genotypes, and high resolution ultrasound was used to measure brachial artery diameter at rest, after reactive hyperaemia and after sublingual glyceryltrinitrate.

Results. The flow-mediated arterial dilation among the subjects with $e 4 / 3$ or $e 4 / 4$ was $3.14 \pm 0.32 \%$, which was lower than that in subjects with $e 2 / 2$ or $e 3 / 2$
\end{abstract}

Apolipoprotein E (Apo E) is one of the major proteins involved in catabolism of triglyceride-rich lipoproteins (VLDL) and remnants. ApoE acts as ligand for two receptors: the "remnants" receptor and the Apo

Received: 29 July 2002 / Revised: 12 November 2002

Published online: 25 March 2003

(C) Springer-Verlag 2003

Corresponding author: X. Guangda, Department of Endocrinology, Wuhan General Hospital, Wu Luo Road 627, Wuhan, 430070 People's Republic of China

E-mail: Guangda64@163.net

Abbreviations: ApoE, Apolipoprotein E; PPBG, postprandial blood glucose; ApoA1, apolipoprotein A1; ApoB, apolipoprotein B; FBG, fasting blood glucose; Lp(a), lipoprotein(a); GNT, glyceryltrinitrate.
$(4.04 \pm 0.30 \%) \quad(p=0.038)$. The baseline vessel size, glyceryltrinitrate-induced arterial dilation and baseline flow were not different among different ApoE genotypes. On univariate analysis, reduced flow-mediated arterial dilation was related to total cholesterol, LDL, lipoprotein(a) [Lp(a)], high blood pressure, older age, family history of premature vascular disease, larger vessel size, cigarette smoking, duration of diabetes and $e 4$ allele $(p<0.05)$. By multiple stepwise regression analysis, reduced flow-mediated arterial dilation was associated with cigarette smoking, LDL, Lp(a), and $e 4$ allele $(p<0.01)$.

Conclusion/interpretation. Apo $e 4$ allele is associated with impairment of endothelium-dependent arterial dilation in the early stage of Type 2 diabetes. [Diabetologia (2003) 46:514-519]

Keywords Type 2 diabetes, endothelial dysfunction, apolipoprotein E genotypes.
$\mathrm{B} / \mathrm{E}$ receptor [1]. In humans, the gene locus for ApoE have three common alleles, $e 2, e 3$, and $e 4$, which encode three major isoforms, e2, e3 and e4, yielding six common genotypes, e2/2,e4/2,e3/2,e3/3,e4/3 and $e 4 / 4$ [1].

In non-diabetic population, many studies $[2,3,4,5$, 6] showed that the Apo $e 4$ allele is associated with the risk of myocardial infarction, the increased severity of coronary artery disease defined by angiography, as well as the increased occurrence of exercise-induced silent myocardial ischaemia. Recently, several studies showed that Apo $e 4$ allele is also associated with the risk of coronary artery disease $[7,8]$ and the increased occurrence of exercise-induced silent myocardial ischaemia [9] in Type 2 diabetic patients. To date, no data are available on the role of ApoE genotypes in endo- 
thelial dysfunction. Endothelial dysfunction is an early physiological event in atherosclerosis [10]. Therefore, we hypothesized that $e 4$ allele is also a risk factor for endothelial dysfunction. The purpose of this study was to investigate the relationship between the Apo e4 allele and endothelial dysfunction in Type 2 diabetes mellitus without clinically detectable angiopathy.

\section{Subjects and methods}

Subjects. The study population included 255 Chinese Han Type 2 diabetic men referred to our hospital, between 40 to 68 years of age, mean age $58 \pm 8$ years. Those patients with microangiopathy and macroangiopathy including nephropathy (urinary albumin excretion rate $>20 \mu \mathrm{g} / \mathrm{min}$ ), retinopathy (at least one microaneurysm or haemorrhage or exudates in either eye), neuropathy (pain in extremities, paresthesias and absent tendon reflexes and/or absent vibration sense), coronary artery disease (myocardial infarction, ischaemia, ECG changes and angina), cerebrovascular disease (transient ischaemic attack or stroke), and peripheral vascular disease (intermittent claudication), and with hypertension were excluded. Each subject was asked details of his smoking history and family history of premature vascular disease. Cigarette smokers were defined as subjects who had smoked at least one cigarette daily for 1 year. Family history was considered positive if a first-degree relative had had clinical evidence of coronary artery disease (angina, myocardial infarction or bypass surgery) at a younger age than 55 years. All the subjects gave informed consent and volunteered for the study which was approved by the Wuhan General Hospital ethics committee.

Methods. Venous blood samples were drawn after a 12- to 14-h overnight fast. Postprandial 2-h blood samples (during an OGTT) were drawn for postprandial blood glucose (PPBG). Measurement of serum lipids, lipoproteins and other parameters, serum total cholesterol, LDL, triglyceride, HDL, were measured enzymatically. Apolipoprotein A1 (ApoA1) and ApoB were measured by immunoturbidimetry. Serum glucose, including fasting blood glucose (FBG) and PPBG were measured by a glucose oxidase procedure. Serum lipoprotein (a) $[\mathrm{Lp}(\mathrm{a})]$ concentrations were measured by an ELISA method. Haemoglobin $\mathrm{A}_{1 \mathrm{c}}$ was measured by high-performance chromatography. Coefficients of variation for these assays were 1 to $2 \%$ (glucose), 1 to $2 \%$ (total cholesterol), 2 to $3 \%$ (LDL), 1 to $2 \%$ (HDL), 2 to $4 \%\left(\mathrm{ApoA}_{1}\right.$ and ApoB), 4 to $7 \%$ [Lp(a)], and 1 to $2 \%$ (haemoglobin $\mathrm{A}_{1 \mathrm{c}}$ ).

The vascular studies of the brachial artery were done noninvasively, as described previously [11]. High resolution ultrasound was used to measure changes in arterial diameter in response to reactive hyperaemia (with increased flow producing an endothelium-dependent stimulus to vasodilation) and to glyceryltrinitrate (GNT, an endothelium-independent vasodilator) (128XP/10 with a 7.0 MHZ linear array transducer: Acuson, Mountain View, Calif., USA).

The subjects rested in the supine position for $10 \mathrm{~min}$ before the first scan and remained supine throughout the study. The target artery (the brachial $2-15 \mathrm{~cm}$ above the elbow) was scanned in longitudinal section and the center of the vessel was identified when the clearest images of anterior and posterior walls of the artery were obtained. The transmit zone was set to the level of the anterior vessel wall. Depth and gain settings were optimized to identify the lumen to vessel wall interface.
Images were magnified with the resolution box function leading to a television line width of $\sim 0.05 \mathrm{~mm}$. Machine settings were kept constant during each study.

Flow increase was induced by inflation of a blood pressure tourniquet placed around the forearm distal to the target artery, to $300 \mathrm{mmHg}$. The cuff was released after 4 to $5 \mathrm{~min}$ and after cuff deflation the artery was scanned continuously for $90 \mathrm{~s}$. A total of 15 min was allowed for vessel recovery, then sublingual GNT (400- $\mu$ g spray) was administered, and 4 to $5 \mathrm{~min}$ later the last scan was done. The electrocardiogram was monitored continuously.

Vessel diameter was measured by two observers, unaware of clinical details and the stage of the experiment. The arterial diameter was measured at a fixed distance from an anatomical maker, such as a bifurcation, with ultrasonic calipers. Measurements were taken from the anterior to the posterior " $\mathrm{m}$ " line at end diastole, incident with the R-wave on the electrocardiogram. The mean diameter was calculated from four cardiac cycles. For the hyperaemia scan, vessel diameter was measured 45 to $60 \mathrm{~s}$ after cuff release. Diameter changes were derived as percent change relative to the first baseline scan $(100 \%)$. Baseline blood flow (measured during the first baseline scan) was estimated by multiplying angle-corrected, pulsed Doppler recordings of the flow-velocity integral by JI and the square of the radius of the artery.

ApoE genotyping, Genomic DNA was extracted from mouthwash water [12]. ApoE genotypes were analysed using polymerase chain reaction ( PCR) or allele-specific oligonucleotide probe technique according to our previous report [9]. PCR was used to amplify a $330 \mathrm{bp}$ region spanning both $e 2 / e 3$ and e3/e4 allelic sites [13]. PCR primers used were $\mathrm{P}_{1} 5^{\prime}$ TCCAAGGAG CTGCAGGCGGCGCA-3', and $\mathrm{P}_{2}$ 5'-TAGCGGCTGGCCGGCCAGGGAG-3'. The allele-specific probes used to detect variation at amino acid 158 were A8 $5^{\prime}$-CTGCAGAAGCGCCTGGCAGTG-3' e3 (e3) ASO and C8 5'-CACTGCCAGGCACTTCTGCAG-3' e2 (e2) ASO, and at amino acid 112 were A2 5'-GAGGACGTGCGCGGCCGCCTG-3' e4 (e4) ASO, and C2 5'-CAG GCGGCCGCACACGTCCTC -3' e3 (e3) ASO [13].

Statistical methods. The number of a given allele, e.g., e3, in the pair of alleles can be 0,1 , or 2 , corresponding to individual relative frequencies of $0 \%, 50 \%, 100 \%$, respectively. A carrier of a specific $e$ allele was a subject carrying the $e 4, e 3$, or $e 2$ genotype in homo- or heterozygous form. Comparison between more than two groups was done by the Chi square test with Yate correction or the ANOVA. Comparison between two groups was done by the Chi square test or Student's $t$ test for independent samples when appropriate. Univariate analysis of the effects of each potential risk factor on flow-mediated arterial dilation and nitroglycerin response was done with linear regression for continuous variables (total cholesterol, LDL, HDL, triglycerides, Lp(a), ApoA1, ApoB, mean blood pressure, age, duration of diabetes, vessel size, blood flow and with one-way analysis of variance for categoric variables (family history, smoking, $e 2, e 3$, and $e 4$ ). The relation between risk factors and flow-mediated dilation or nitroglycerin was then examined using multiple stepwise regression analysis. Lp(a) concentrations were log-transformed before analysis. All analyses were carried out by using the statistical package SAS. $e 4 / 2$ was excluded from the analysis because of the very low prevalence and the contrary effect on serum lipids between $e 4$ and $e 2$. 
Table 1. Serum lipid lipoprotein and apolipoprotein concentrations in Type 2 diabetes by ApoE genotypes

\begin{tabular}{lccr}
\hline & $e 2 / 2 e 3 / 2(n=34)$ & $e 3 / 3(n=161)$ & $e 4 / 3 e 4 / 4(n=58)$ \\
\hline Total cholesterol (mmol/l) & $5.11 \pm 1.13$ & $5.37 \pm 1.15$ & $5.94 \pm 1.26^{\mathrm{a}}$ \\
LDL (mmol/l) & $2.38 \pm 0.97$ & $2.57 \pm 1.07$ & $3.22 \pm 1.01^{\mathrm{a}}$ \\
Triglyceride (mmol/l) & $3.09 \pm 1.12$ & $2.68 \pm 1.20$ & $2.42 \pm 1.22$ \\
HDL (mmol/l) & $1.13 \pm 0.17$ & $1.14 \pm 0.23$ & $1.11 \pm 0.20$ \\
ApoA $(\mathrm{g} / \mathrm{l})$ & $1.21 \pm 0.21$ & $1.19 \pm 0.30$ & $1.22 \pm 0.18$ \\
ApoB (g/l) & $1.12 \pm 0.24$ & $1.10 \pm 0.27$ & $1.09 \pm 0.25$ \\
Lp(a) (mg/l) & $133 \pm 56^{\mathrm{b}}$ & $328 \pm 124$ & $480 \pm 187$ \\
\hline
\end{tabular}

a $p<0.05$ compared with $e 2 / 2 e 3 / 2$

${ }^{\mathrm{b}} p<0.05$ compared with other two groups

Table 2. Clinical characteristics and metabolic control for Type 2 diabetes by ApoE genotypes

\begin{tabular}{lccc}
\hline & $e 2 / 2 e 3 / 2(n=34)$ & $e 3 / 3(n=161)$ & $e 4 / 3 e 4 / 4(n=58)$ \\
\hline Age (years) & $59 \pm 7$ & $58 \pm 10$ & $57 \pm 9$ \\
Duration of diabetes (years) & $5 \pm 2$ & $6 \pm 3$ & $6 \pm 2$ \\
BMI (Kg/m ${ }^{2}$ ) & $24.5 \pm 0.54$ & $24.0 \pm 0.76$ & $23.9 \pm 0.66$ \\
Positive family history $[n(\%)]$ & $3(8.82)$ & $17(10.56)$ & $8(13.79)$ \\
Cigarette smoking $[n(\%)]$ & $8(23.53)$ & $38(23.60)$ & $15(25.86)$ \\
Mean blood pressure (mmHg) & $96 \pm 29$ & $100 \pm 48$ & $105 \pm 37$ \\
FBG (mmol/l) & $7.6 \pm 2.4$ & $8.0 \pm 2.7$ & $7.8 \pm 3.0$ \\
PPBG (mmol/l) & $11.3 \pm 4.5$ & $11.5 \pm 5.1$ & $12.2 \pm 4.7$ \\
Haemoglobin $A_{1 \mathrm{C}}(\%)$ & $8.2 \pm 2.1$ & $8.0 \pm 2.3$ & $8.3 \pm 2.3$ \\
\hline
\end{tabular}

Table 3. The results of brachial artery studies by ApoE genotypes

\begin{tabular}{lccc}
\hline & $e 2 / 2 e 3 / 2(n=34)$ & $e 3 / 3(n=161)$ & $e 4 / 3 e 4 / 4(n=58)$ \\
\hline Baseline vessel (mm) & $3.74 \pm 0.55$ & $3.77 \pm 0.42$ & $3.72 \pm 0.61$ \\
Flow-mediated dilation (\%) & $4.04 \pm 0.30$ & $3.71 \pm 0.49$ & $3.41 \pm 0.32 \mathrm{a}$ \\
GNT-induced dilation (\%) & $20.22 \pm 1.41$ & $19.56 \pm 1.33$ & $20.37 \pm 1.27$ \\
Baseline flow (ml/min) & $81.88 \pm 37.90$ & $82.54 \pm 40.53$ & $83.33 \pm 36.44$ \\
\hline
\end{tabular}

a $p<0.05$ compared with $e 2 / 2 e 3 / 2$ group

\section{Results}

The ApoE genotype prevalence was as follows: $1.6 \%$ for $e 2 / 2,0.8 \%$ for $e 4 / 2,11.8 \%$ for $e 3 / 2,63.1 \%$ for $e 3 / 3,19.6 \%$ for $e 4 / 3$, and $3.1 \%$ for $e 4 / 4$. The observed distribution of ApoE genotypes was in Hardy-Weinberg equilibrium. Serum total cholesterol and LDL concentrations were higher in subjects with $e 4 / 3$ or $e 4 / 4$ than in subjects with $e 2 / 2$ or $e 3 / 2(p=0.021)$. $\mathrm{Lp}$ (a) concentration was lower in subjects with $e 2 / 2$ or $e 3 / 2$ than in subjects with $e 3 / 3$ and $e 4 / 3$ or $e 4 / 4$ $(p=0.043)$. Other serum lipid and lipoprotein concentrations did not differ among the ApoE genotypes (Table 1). With respect to clinical characteristics and metabolic control of diabetes, the ApoE genotype groups did not differ from each other (Table 2).

Table 3 shows the results of brachial artery studies by ApoE genotypes. The flow-mediated arterial dilation among the subjects with genotype $e 4 / 3$ or $e 4 / 4$ was $3.14 \pm 0.32 \%$, which was lower than those in sub- jects with $e 2 / 2$ or $e 3 / 2(4.04 \pm 0.30 \%)(p=0.038)$. The baseline vessel size (diameter), GNT-induced arterial dilation and baseline flow were not significantly different among different ApoE genotypes.

Univariate analysis showed a correlation between flow-mediated arterial dilation and high total cholesterol concentration $(r=-0.23, p<0.001)$, high LDL $(r=-0.26, p<0.001)$, high blood pressure $(r=-0.30$, $p<0.001)$, positive family history $(r=-0.16, p=0.03)$, older age $(r=-0.14, p=0.005)$, larger vessel size $(r=-$ $0.31, p<0.001)$, duration of diabetes $(r=-0.25$, $p=0.0003)$, cigarette smoking $(r=-0.33, p<0.001)$, $\mathrm{Lp}(\mathrm{a})(r=-0.33, p<0.001)$ and $e 4$ allele $(r=-0.38$, $p<0.001)$. On stepwise multiple regression analysis, flow-mediated arterial dilation was negatively correlated with older age, larger vessel size, LDL, Lp(a), Apo $e 4$, duration of diabetes, cigarette smoking and positive family history $(p<0.01)$ (Table 4$)$, but not with total cholesterol, triglyceride, HDL and blood pressure. 
Table 4. Stepwise multiple regression analyses on flow mediated dilation with age, vessel size, total cholesterol, triglyceride, HDL, LDL, Lp(a), Apo e4, duration of diabetes, cigarette smoking, family history, blood pressure as predictors

\begin{tabular}{|c|c|c|c|c|c|c|}
\hline Variable & B & $\mathrm{SE}_{\mathrm{B}}$ & $t$ & $p$ value & $r^{2}$ & $\mathrm{~F}(\mathrm{p})$ \\
\hline Older age & -0.028 & 0.003 & 19.43 & 0.0001 & & \\
\hline Larger Vessel size & -0.35 & 0.101 & 16.14 & 0.0003 & & \\
\hline $\mathrm{Lp}(\mathrm{a})$ & -0.20 & 0.222 & 25.10 & 0.0000 & & \\
\hline Apo $e 4$ & -0.41 & 0.243 & 21.37 & 0.0000 & & \\
\hline Duration of diabetes & -0.25 & 0.064 & 5.88 & 0.001 & & \\
\hline Constant & 9.59 & 0.284 & 28.72 & 0.0000 & 0.44 & $66.82(0.0000)$ \\
\hline
\end{tabular}

$\mathrm{B}$, partial regression coefficient; $\mathrm{SE}_{\mathrm{B}}$, standard error of $\mathrm{B} ; t$, Student's $t$ test; $r^{2}$, coefficient of determination; $\mathrm{F}(\mathrm{p})$, results from F-test of model and $p$ values of the tests. Smoking and family history were entered as categoric variables (nonsmok-

\section{Discussion}

Endothelial dysfunction is an important early event in atherogenesis $[14,15]$. Both spatial and temporal correlations between endothelial dysfunction and coronary atherosclerosis have been shown in animal models and in humans $[16,17,18]$. Impairment of endothelial function in early life could result in abnormal reactions between the vessel wall and platelets, neutrophils, and macrophages, and thus could contribute to the initial stages of atherogenesis [14]. However, the underlying mechanisms of the relationship between Apo e 4 allele and the impairment of endothelial function are not clear. Our subjects were Type 2 diabetic men without clinically detectable angiopathy and hypertension, indicating they are in the early stage of diabetes. No significant differences on clinical characteristics, such as age, BMI, blood pressure, blood sugar, cigarette smoking, duration of diabetes, family history, were found among different ApoE genotype groups, indicating that susceptibility to impairment of endothelium-dependent arterial dilation cannot be explained by classic risk factors alone [19, 20]. Endothelial injury has been induced experimentally by hypercholesterolaemia, particularly the LDL component [21]. In clinical studies using invasive techniques, hypercholesterolaemic adults have been shown to have impaired endothelium-dependent responses to a variety of pharmacologic stimuli, including infusions of acetyleholine [22,23]. Recently, another study using a noninvasive method, showed that impaired endothelium-dependent arterial dilation is present in children with familial hypercholesterolaemia as young as 7 years of age [24]. In the present study, compared with subjects with $e 2$ allele, the subjects with $e 4$ allele have higher total cholesterol, and LDL concentrations. Flow-mediated arterial dilation was correlated with LDL and $e 4$ allele, which are in good agreement with previous studies $[2,3,4,5,6,7$, $8,9]$. These can explain partially the impairment of ing $=0$, smoking $=1$, and negative family history $=0$, positive history=1). Allele types are coded 0 if not present in the pair of alleles, 0.5 if one allele is present, and 1 if the specific allele is present in both members of the pair

endothelial-dependent arterial dilation in people with the $e 4$ allele.

$\mathrm{Lp}(\mathrm{a})$ is an independent risk factor for atherosclerotic vascular disease [25, 26]. Many prospective studies have shown that excess $L p(a)$ is associated with premature coronary atherosclerosis and risk of cardiovascular disease [27, 28]. Moreover, increased plasma $\mathrm{Lp}(\mathrm{a})$ concentrations have been associated with subclinical carotid atherosclerosis in patients with Type 2 diabetes mellitus in some studies [29], but not all [30]. The mechanism by which high concentrations of $\mathrm{Lp}(\mathrm{a})$ are related to atherosclerosis is not clear, but $\mathrm{Lp}(\mathrm{a})$ could interfere with intravascular thrombolysis and inhibit the streptokinaze-mediated conversion of plasmin from plasminogen. In addition, $\mathrm{Lp}(\mathrm{a})$ is found within atherosclerotic plaque and could contribute to cholesterol ester accumulation within the plaque [31]. In 1994, a relation between $\mathrm{Lp}$ (a) and impaired endothelium-dependent arterial dilation in 1 to 17 years old children with familial hypercholesterolaemia, independent of the total cholesterol concentration was reported [24]. More recently, a study showed that increased $\mathrm{Lp}(\mathrm{a})$ concentrations are associated with an impaired endothelial function in healthy postmenopausal women, independent of conventional risk factors for cardiovascular disease [32]. Our data showed a negative relation between plasma $\mathrm{Lp}$ (a) concentrations and endothelium-dependent arterial dilation, and the $\operatorname{Lp}(\mathrm{a})$ concentrations in subjects with $e 2 / 2$ or $e 3 / 2$ were lower than those in subjects with $e 3 / 3$ as well as subjects with $e 4 / 3$ or $e 4 / 4$. These can also explain partially the impairment of endotheliumdependent arterial dilation in people with the $e 4$ allele in the early stage of Type 2 diabetes mellitus.

Smoking has long been recognised as one of the major risks for vascular disease. It is associated with severe aortic atherosclerosis in patients over 25 years of age [33] and with early lesions in patients younger than 25 years [34]. Previously, several studies showed that cigarette smoking is associated with impaired 
endothelium-dependent arterial dilation in healthy people [11, 19, 32, 35]. Furthermore, exposure to heavy environmental tobacco smoke (passive smoking) can also cause impairment of endothelium-dependent arterial dilation. We also found simillar results in the early stage of Type 2 diabetes mellitus in men. In addition, aging and a positive family history of premature vascular disease are two of the risk factors that are correlated with endothelial dysfunction [19], this association was detected in our study as well.

In summary, these results showed an association between the Apo $e 4$ allele and endothelium-dependent arterial dilation in the early stage of Type 2 diabetes mellitus, although the mechanism for the association has not been explained completely.

\section{References}

1. Davignon J, Gregg RE, Sing CF (1988) Apolipoprotein E polymorphism and atherosclerosis. Atherosclerosis 8:1-21

2. Luc G, Bard JM, Arveiler D et al. (1994) Impact of apolipoprotein $\mathrm{E}$ polymorphism on lipoproteins and risk of myocardial infarction: The ECTIM Study. Arterioscler Thromb 14:1412-1419

3. Tirek L, Menzel HJ, Ehnholm C, Nicaud V, Havekes LM (1994) ApoE polymorphism and predisposition to coronary heart disease in youth of different European populations: The EARS Study. European Atherosclerosis Research Study. Arterioscler Thromb 14:1617-1624

4. Wang XL, McCredie RM, Wilcken DEL (1995) Polymorphisms of the apolipoprotein E gene and severity of coronary artery disease defined by angiography. Arterioscler Thromb Vasc Biol 15:1030-1034

5. Katzel LI, Fleg JL, Paidi M, Ragoobarsingh N, Goldberg AP (1993) ApoE $_{4}$ polymorphism increases the risk for exercise-induced silent myocardial ischemia in older men. Arterioscler Thromb 13:1495-1500

6. Nakata Y, Katsuya T, Rakugi H et al. (1996) Polymorphism of the apolipoprotein $\mathrm{E}$ and angiotensin-converting enzyme genes in Japanese subjects with silent myocardial ischemia. Hypertension 27:1205-1209

7. Laakso M, Kesäniemi YA, Kervinen K, Jauhiainen M, Pyörälä K (1991) Relationship of coronary heart disease and apolipoprotein $\mathrm{E}$ phenotype in patients with non-insulindependent diabetes. BMJ 303:1159-1162

8. Ukkola O, Kervinen K, Salmela PI, Dickhoff KC, Laakso M, Kesäniemi YA (1993) Apolipoprotein E phenotype is related to macro- and microangiopathy in patients with non-insulin dependent diabetes mellitus. Atherosclerosis 101:9-15

9. Guangda X, Bangshun X, Xiujian L, Yangzhong H (1999) Apo e4 allele increases the risk for exercise-induced silent myocardial ischemia in non-insulin dependent diabetes mellitus. Atherosclerosis147:293-296

10. Healy B (1990) Endothelial cell dysfunction: an emerging endocrinopathy linked to coronary disease. J Am Coll Cardiol 16:357T-358T

11. Celermajier DS, Soerensen KE, Gooch VM et al. (1992) Non-invasive detection of endothelial dysfunction in children and adults at risk of atherosclerosis. Lancet 340:1111-1115

12. Bolla MK, Haddad L, Homphries SI (1995) High throughput method for determination of apolipoprotein E genotypes with use of restriction digestion analysis by microplate array diagonel gel electrophoresis. Clin Chem 41:1600-1606

13. Houlston RS, Snowden C, Green F, Alberti KGMN, Humphries SE (1989) Apolipoprotein(apo) E genotypes by polymerase chain reaction and allele specific oligonucleotide probes: no detectable linkage disequilibrium between ApoE and ApoCII. Hum Genet 83:364-368

14. Ross R (1986) The pathogenesis of atherosclerosis, an update. N Engl J Med 8:488-500

15. Henderson AH (1991) Endothelium in control. Br Heart J 65:116-125

16. Mclenacham JM, Vita JA, Fish RD et al. (1990) Early evidence of endothelial dysfunction at coronary branchpoints. Circulation 82:1169-1173

17. Harrison DG, Armstrong MI, Freman PC, Heistad DD (1987) Restoration of endothelium-dependent relaxation by dietary treatment of atherosclerosis. J Clin Invest 80:1808-1811

18. Fish RD, Nabel EG, Selwyn AP et al. (1988) Responses of coronary arteries of cardiac transplant patients to acetylcholine. J Clin Invest 81:21-31

19. Celermajer DS, Sorensen KE, Bull C, Robinson J, Deanfield JE (1994) Endothelium-dependent dilation in the systemic artery of asymptomatic subjects relates to coronary risk factors and their interaction. J Am Coll Cardiol 24:1468-1474

20. Vehkavaara S, Groop PH, Seppala-Lindroos A, Yki-Jarvinen $\mathrm{H}$, Westerbaeba J (1999) In vivo endothelial dysfunction characterizes patients with impaired fasting glucose. Diabetes Care 22:2055-2060

21. Galle J, Bassenge E, Busse R (1990) Oxidized low-density lipoproteins potentiate vasoconstrictions to various agonists by direct interaction with vascular smooth muscle. Circ Res 66:1287-1293

22. Drexler H, Zeiher AM, Memzer K, Just H (1991) Correction of endothelial of dysfunction in coronary microcirculation of hypercholesterolemic patients by L-argmine. Lancet 338:1546-1550

23. Vitu JA, Ireasure CB, Nabel IG et al. (1981) Coronary vasomotor response to acetylcholine relates to risk factors for coronary artery disease. Circulation 81:491-497

24. Sorensen KE, Celermajier DS, Georgakopoulos D, Hatcher G, Betteridge DJ, Deanfield JE (1994) Impairment of endothelium-dependent dilation is an early event in children with familial hypercholesterolemia and is related to the lipoprotein (a) level. J Clin Invest 93:50-55

25. Maher VMG, Brown BG (1995) Lipoprotein(a) and coronary heart disease. Curr Opin Lipidol 6:229-235

26. Mijatovic V, vander Mooren MJ, Stehouwer CDA, Netelenbos JC, Kenemans P (1999) Postmenopausal hormone replacement, risk estimations for coronary artery disease and cardiovascular protection. Gynecol Endocrinol 13:130-144

27. Skin JH, Rosenson RS (1997) Lipoprotein Lp(a) excess and coronary heart disease. Arch Intern Med 157:1170-1176

28. Genest J Jr, McNamara JR, Ordovas JM et al. (1992) Lipoprotein cholesterol, Apolipoprotein A-I and B and lipoprotein(a) abnormalities in men with premature coronary artery disease. J Am Coll Cardiol 19:792-802

29. Yamamoto M, Egusa G, Yamakido M (1997) Carotid atherosclerosis and serum lipoprotein(a) concentrations in patients with NIDDM. Diabetes Care 20:829-831

30. Yamasaki Y, Kawamori R, Matsushima H et al. (1994) Atherosclerosis in carotid artery of young IDDM patients monitored by ultrasound high-resolution B-mode imaging. Diabetes 43:634-639

31. Utermann G (1989) The mysteries of lipoprotein(a). Science 246:904-1000 
32. Wilmink HW, de Kleijn MJJ, Bots ML et al. (2000) Lipoprotein(a) is associated with endothelial function in healthy postmenopausal women. Atherosclerosis 153: 249-254

33. McGill HC Jr (1988) The cardiovascular pathology of smoking. Am Heart J 115:250-257

34. McGill HC Jr (1990) Relationship of atherosclerosis in young men to serum lipoprotein cholesterol concentration and smoking: a preliminary report from the Pathobiological Determinants of Atherosclerosis in Youth (PDAY) research group. JAMA 264:3018-3024

35. Celermajer DS, Sorensen KE, Georgakopoulos D et al. (1993) Cigarette smoking is associated with dose-related and potentially reversible impairment of endothelium-dependent dilation in healthy young adults. Circulation $88: 2149-2155$ 